\title{
A NOTE ON LOCAL CHANGE OF DIFFEOMORPHISM
}

\author{
MIKIYA MASUDA
}

\begin{abstract}
Let $D(M)$ be the group of pseudo-isotopy classes of orientation preserving diffeomorphisms of a compact manifold $M$ which restrict to the identity on $\partial M$. If a compact manifold $N$ of the same dimension as $M$ is embedded in $M$, then extending maps in $D(N)$ as the identity on the exterior of $N$ defines a homomorphism $E: D(N) \rightarrow D(M)$. We ask if the kernel of $E$ is finite and show that this is the case for special cases.
\end{abstract}

\section{INTRODUCTION}

Two diffeomorphisms $f_{0}$ and $f_{1}$ of a smooth manifold $M$, which are the identity on the boundary $\partial M$ of $M$ if $\partial M$ is nonempty, are called pseudoisotopic (or concordant) if there is a diffeomorphism $F$ of $M \times I \quad(I=[0,1])$ such that $F(x, 0)=\left(f_{0}(x), 0\right), F(x, 1)=\left(f_{1}(x), 1\right)$ for all $x \in M$ and that $F$ is the identity on $\partial M \times I$. The set $D(M)$ of pseudo-isotopy classes of orientation preserving diffeomorphisms of $M$ forms a group under composition of maps.

It is interesting by itself to compute $D(M)$ and moreover it is sometimes related to geometrical problems, e.g. when $M=D^{n}$ or $S^{n}[\mathrm{KM}], S^{p} \times S^{q}$ [B, L], $\mathbf{C} P^{k} \times D^{q}$ [BP]. The group $D(M)$ is well understood for some $M$, but in general it seems not so well understood. For instance, the author does not know a higher dimensional example of $M$ such that $D(M)$ is trivial.

Let $N$ be a compact manifold embedded in $M$ of the same dimension. Then there is defined a homomorphism $E: D(N) \rightarrow D(M)$ by extending a map in $D(N)$ as the identity on the exterior of $N$. Then it is natural to ask

Question. Is ker $E$ trivial or finite?

An interesting case is when $N$ is an $n$-disk $D^{n}$. As is well known $D\left(D^{n}\right)$ is isomorphic to the Kervaire-Milnor group $\theta_{n+1}$ of oriented homotopy $(n+1)$ spheres $(n \geq 4)$ and the group is nontrivial in general if $n \geq 6$. Thus if $\operatorname{ker} E$ is trivial for $N=D^{n}$, then one can conclude that $D(M)$ is nontrivial in general.

In this paper we consider the case where $N=\mathbf{C} P^{k} \times D^{q}$. The group $D\left(\mathbf{C} P^{k} \times D^{q}\right)$ is fairly well understood by Browder-Petrie [BP] in connection

Received by the editors September 14, 1987.

1980 Mathematics Subject Classification (1985 Revision). Primary 57R50; Secondary 57S15. 
with the study of semifree circle actions on homotopy spheres. The purpose of this paper is to prove the following, which gives an evidence supporting the above question.

Theorem A. Let $X$ be a closed orientable manifold of dimension $q \geq 2$ such that $H^{1}(X ; \mathbf{Z})=0$. Then the kernel of

$$
E=E_{X}: D\left(\mathbf{C} P^{k} \times D^{q}\right) \rightarrow D\left(\mathbf{C} P^{k} \times X\right)
$$

is finite, where $D^{q}$ is any q-disk embedded in $X$.

Remark. The map $E_{X}$ depends on the choice of an embedding of $D^{q}$ into $X$ in general. However the disk theorem tells us that it only depends on a connected component of $X$ into which $D^{q}$ is embedded and on whether the embedding preserves orientation or not (we fix an orientation on $D^{q}$ ).

The group $D\left(\mathbf{C} P^{k} \times D^{q}\right)$ is finitely generated abelian. The rank $r_{k, q}$ of the free part is explicitly computed [BP]. In fact, if $q$ is even $r_{k, q}=0$, i.e. $D\left(\mathbf{C} P^{k} \times D^{q}\right)$ is finite; so Theorem A is trivial in this case. If $q$ is odd, $r_{k, q}$ is nonzero in most cases. In fact, it is given by

$$
r_{k, q}=[k / 2]+a_{k, q}+b_{k, q}
$$

where

$$
\begin{aligned}
& a_{k, q}= \begin{cases}1 & \text { if } k \text { is odd and } q+1 \equiv 0(4), \\
0 & \text { otherwise },\end{cases} \\
& b_{k, q}= \begin{cases}1 & \text { if } 3 \leq q \leq 2 k+1(q: \text { odd }), \\
0 & \text { otherwise. }\end{cases}
\end{aligned}
$$

Let $\operatorname{Diff}_{+} M$ be the group (with $C^{\infty}$ topology) of orientation preserving diffeomorphisms of $M$ which restrict to the identity on $\partial M$. The connected components $\pi_{0}\left(\operatorname{Diff}_{+} M\right)$ are nothing but the isotopy classes of those diffeomorphisms; so there is a natural epimorphism $\Pi: \pi_{0}\left(\operatorname{Diff}_{+} M\right) \rightarrow D(M)$.

Similarly to $E_{X}$, a homomorphism

$$
E_{X}^{\prime}: \pi_{0}\left(\operatorname{Diff}_{+} \mathbf{C} P^{k} \times D^{q}\right) \rightarrow \pi_{0}\left(\operatorname{Diff}_{+} \mathbf{C} P^{k} \times X\right)
$$

is defined. Clearly $\Pi$ commutes with $E_{X}$ and $E_{X}^{\prime}$. According to Cerf [C] $\Pi: \pi_{0}\left(\mathrm{Diff}_{+} \mathbf{C} P^{k} \times D^{q}\right) \rightarrow D\left(\mathbf{C} P^{k} \times D^{q}\right)$ is an isomorphism (when $\operatorname{dim} \mathbf{C} P^{k} \times$ $\left.D^{q}=2 k+q \geq 5\right)$ because $\mathbf{C} P^{k} \times D^{q}$ is simply connected. Hence we have

Corollary B. Let $X$ be the same as in Theorem A. Then the kernel of $E_{X}^{\prime}$ is finite when $2 k+q \geq 5$.

The outline of the proof of Theorem A is as follows. First, by using the Atiyah-Singer invariant [AS, §7], we define an invariant $\sigma$ on a subgroup of $D\left(\mathbf{C} P^{k} \times X\right)$ containing the image of $E_{X}(\S 1)$. Next we see that the composition $\sigma \cdot E_{X}$ is independent of $X$ (Lemma 2.1). Thirdly we see that $\sigma \cdot E_{X}$ is a homomorphism (Lemma 3.1). Finally we check the finiteness of the kernel of 
$\sigma \cdot E_{X}$ for $X=S^{q}$ (Theorem 4.1). The assumption $H^{1}(X ; \mathbf{Z})=0$ is used for the well-definedness of the invariant $\sigma$.

\section{AN INVARIANT $\sigma$}

As remarked in the introduction Theorem A is trivial when $q=\operatorname{dim} X$ is even. Therefore $q$ will be odd throughout this paper unless otherwise stated.

We orient $X$. The disjoint union $X \amalg-X$ ( - denotes the reversed orientation) is null-cobordant in the oriented cobordism. Extending maps in $D\left(\mathbf{C} P^{k} \times X\right)$ as the identity on $\mathbf{C} P^{k} \times(-X)$ induces a monomorphism

$$
D\left(\mathbf{C} P^{k} \times X\right) \rightarrow D\left(\mathbf{C} P^{k} \times(X \amalg-X)\right) .
$$

Thus it suffices to prove Theorem A for $X$ being null-cobordant.

Let $\alpha$ be a generator of $H^{2}\left(\mathbf{C} P^{k} ; \mathbf{Z}\right)$ and we also regard $\alpha$ as an element of $H^{2}\left(\mathbf{C} P^{k} \times X\right)$ via the projection map $\mathbf{C} P^{k} \times X \rightarrow \mathbf{C} P^{k}$.

Definition. $D_{0}\left(\mathbf{C} P^{k} \times X\right)=\left\{[f] \in D\left(\mathbf{C} P^{k} \times X\right) \mid f^{*} \alpha=\alpha\right\}$, where $[f]$ denotes the class of a diffeomorphism $f$ of $\mathbf{C} P^{k} \times X$.

Clearly $D_{0}\left(\mathbf{C} P^{k} \times X\right)$ forms a subgroup of $D\left(\mathbf{C} P^{k} \times X\right)$. The image of $E_{X}$ is contained in $D_{0}\left(\mathbf{C} P^{k} \times X\right)$. We shall define an invariant

$$
\sigma: D_{0}\left(\mathbf{C} P^{k} \times X\right) \rightarrow F\left(S^{1}\right) / \mathbf{Z}
$$

where $F\left(S^{1}\right) / \mathbf{Z}$ is the quotient group of $F\left(S^{1}\right)$, the fraction field of the complex character (or representation) ring $R\left(S^{1}\right)$ of the circle group $S^{1}$, divided by the normal subgroup $\mathbf{Z}$ consisting of integer valued constant functions on $S^{1}$. Since $R\left(S^{1}\right)$ is the Laurent polynomial ring $\mathrm{Z}\left[t, t^{-1}\right]$ as is well known, $F\left(S^{1}\right)$ is the field of rational functions of $t$.

Let $Y$ and $Y^{\prime}$ be a pair of compact connected oriented manifolds which are bounded by $X$ and have the same signature. Let $[f]$ be an element of $D_{0}\left(\mathbf{C} P^{k} \times X\right)$. We paste together $\mathbf{C} P^{k} \times Y$ and $-\mathbf{C} P^{k} \times Y^{\prime}$ along the boundary by $f$ to get a closed oriented manifold $M$. The oriented diffeomorphism type of $M$ does not depend on the choice of a representative $f$ of $[f]$.

The $S^{1}$ bundle over $\mathbf{C} P^{k} \times X$ corresponding to $\alpha$ is $S^{2 k+1} \times X \rightarrow \mathbf{C} P^{k} \times X$ where $S^{1}$ acts on $S^{2 k+1}$ linearly and freely. Since $f^{*} \alpha=\alpha, f$ lifts to an $S^{1}$ equivariant diffeomorphism $\tilde{f}$ of $S^{2 k+1} \times X$. The difference of two liftings of $f$ is measured by a continuous map from $X$ to $S^{1}$. The homotopy classes of such maps are exactly $H^{1}(X ; \mathbf{Z})$ and we assume the group vanishes. Hence the lifting is unique up to $S^{1}$ equivariant isotopy.

We paste together $S^{2 k+1} \times Y$ and $-S^{2 k+1} \times Y^{\prime}$ along the boundary by $\tilde{f}$ to get a closed oriented manifold $\widetilde{M}=\widetilde{M}\left([f], Y, Y^{\prime}\right)$ with a free $S^{1}$ action. Since $q$ is odd, $\widetilde{M}$ is of odd dimension. Therefore the Atiyah-Singer invariant $\sigma(g, \widetilde{M}) \in \mathbf{C}$ is defined for $g \neq 1 \in S^{1}$. The function $\sigma(, \widetilde{M})$ belongs to the fraction field $F\left(S^{1}\right)$ (see [AS, §7]). Note that the function $\sigma(, \widetilde{M})$ is 
independent of the choice of a representative $f$ because so is the oriented $S^{1}$ diffeomorphism type of $\widetilde{M}$.

Lemma 1.1. The function $\sigma(, \widetilde{M})$, regarded as an element of $F\left(S^{1}\right) / \mathbf{Z}$, depends only on $[f]$, and not on the choice of $Y$ and $Y^{\prime}$.

Proof. Let $A(Y)$ be the closed $S^{l}$ manifold defined as

$$
A(Y)=D^{2 k+2} \times X \cup S^{2 k+1} \times Y
$$

where $D^{2 k+2} \times X$ and $S^{2 k+1} \times Y$ are pasted together along the boundary by $\tilde{f}$. We consider the $S^{1}$ manifold defined as

$$
W=D^{2 k+2} \times Y \cup A(Y) \times I \cup D^{2 k+2} \times Y^{\prime}
$$

where $D^{2 k+2} \times Y$ and $D^{2 k+2} \times Y^{\prime}$ are attached to $A(Y) \times I$ along $D^{2 k+2} \times X$ via the identity map at 0 - and 1-levels respectively. We orient $W$ suitably so that $W$ is an oriented $S^{1}$ cobordism between $\widetilde{M}([f], Y, Y)$ and $\widetilde{M}\left([f], Y, Y^{\prime}\right)$.

By definition we have

$$
\sigma(g, \partial W)=L(g, W)-\operatorname{Sign}(g, W) \text { for } g \neq 1 \in S^{1}
$$

where $L(g, W)$ is the number occurring on the right-hand side of the $G$ signature formula and $\operatorname{Sign}(g, W)$ is the equivariant signature of $W$ evaluated on $g$. Since $S^{1}$ is a connected group $\operatorname{Sign}(g, W)=\operatorname{Sign} W$. As for $L(g, W)$ the $G$-signature formula involves the characteristic classes of $T W^{g}$ and the normal bundle of $W^{g}$, so we have to investigate them. First we note that the $S^{1}$ action on $W$ is semifree, so $W^{g}=W^{S^{1}}$. The fixed point set $W^{S^{1}}$ is $Y \cup X \times I \cup\left(-Y^{\prime}\right)$. The normal bundle to $W^{S^{1}}$ admits a complex structure induced from the $S^{1}$ action. As easily observed the bundle is trivial. Furthermore we have

$$
\text { Sign } W^{S^{1}}=\operatorname{Sign} Y-\operatorname{Sign} Y^{\prime}=0
$$

by the additivity property of signature (see [AS, §7]) and the assumption that Sign $Y=\operatorname{Sign} Y^{\prime}$. Putting these into the $G$-signature formula, one can see that $L(g, W)=0$. Thus we have

$$
\sigma\left(g, \widetilde{M}\left([f], Y, Y^{\prime}\right)\right)-\sigma(g, \widetilde{M}([f], Y, Y))=\sigma(g, \partial W)=-\operatorname{Sign} W
$$

and hence

$$
\sigma\left(, \widetilde{M}\left([f], Y, Y^{\prime}\right)\right)=\sigma(, \widetilde{M}([f], Y, Y)) \text { in } F\left(S^{1}\right) / Z .
$$

Applying the same argument to $\widetilde{M}\left(\left[f^{-1}\right], Y^{\prime}, Y\right)$, we get

$$
\sigma\left(, \widetilde{M}\left(\left[f^{-1}\right], Y^{\prime}, Y\right)\right)=\sigma\left(, \widetilde{M},\left[f^{-1}\right], Y^{\prime}, Y^{\prime}\right) \text { in } F\left(S^{1}\right) / Z \text {. }
$$

Here we note that $\widetilde{M}\left(\left[f^{-1}\right], Y^{\prime}, Y\right)=-\widetilde{M}\left([f], Y, Y^{\prime}\right)$ and $\widetilde{M}\left(\left[f^{-1}\right], Y^{\prime}, Y^{\prime}\right)=$ $-\widetilde{M}\left([f], Y^{\prime}, Y^{\prime}\right)$. Since the Atiyah-Singer invariant changes the sign if the orientation of the manifold is reversed, the above identities prove the lemma. Q.E.D. 
Definition. We set $\sigma([f])(g)=\sigma(g, \widetilde{M}([f], Y, Y))$ and regard $\sigma([f])$ as an element of $F\left(S^{1}\right) / \mathbf{Z}$.

By Lemma $1.1 \sigma([f]) \in F\left(S^{1}\right) / \mathbf{Z}$ is an invariant of $[f] \in D_{0}\left(\mathbf{C} P^{k} \times X\right)$.

2. $\sigma \cdot E_{X}$ IS INDEPENDENT OF $X$

As remarked before the image of $E_{X}: D\left(\mathbf{C} P^{k} \times D^{q}\right) \rightarrow D\left(\mathbf{C} P^{k} \times X\right)$ is contained in $D_{0}\left(\mathbf{C} P^{k} \times X\right)$. Hence the composition $\sigma \cdot E_{X}: D\left(\mathbf{C} P^{k} \times D^{q}\right) \rightarrow F\left(S^{1}\right) / \mathbf{Z}$ is defined. The purpose of this section is to verify

Lemma 2.1. $\sigma \cdot E_{X}$ is independent of $X$.

Proof. It suffices to prove $\sigma \cdot E_{X}=\sigma \cdot E_{S^{q}}$. Let $Y$ be a connected compact oriented manifold bounded by $X$. We may assume that $H^{1}(Y ; \mathbf{Z})=0$, if necessary, by doing surgery to kill the fundamental group of $Y$. Let $\stackrel{\circ}{Y}$ be the cobordism between $X$ and $S^{q}$ obtained by removing a small open disk from $Y$. We choose a smooth simple path in $\stackrel{\circ}{Y}$ connecting $X$ and $S^{q}$ which is transverse to the boundary. The tubular neighborhood is of the form $D^{q} \times I$, where $I$ is the path direction and $D^{q}$ is the normal direction.

Let $[f]$ be an element of $D\left(\mathbf{C} P^{k} \times D^{q}\right)$ and let id be the identity map of $I$. The map $f \times$ id restricts to the identity map on $\mathbf{C} P^{k} \times S^{q-1} \times I$; so it extends to a diffeomorphism of $\mathrm{C} P^{k} \times \stackrel{\circ}{Y}$, say $F$, as the identity on the exterior of $\mathbf{C} P^{k} \times D^{q} \times I$. Since $H^{1}(\stackrel{\circ}{Y} ; \mathbf{Z})=0, F$ lifts uniquely to an $S^{1}$ equivariant diffeomorphism $\widetilde{F}$ of $S^{2 k+1} \times \stackrel{\circ}{Y}$ up to $S^{1}$ equivariant isotopy.

We view $\partial(Y \times I)$ as a triad consisting of three pieces $Y \times\{0\} \cup X \times I \cong Y)$, $\stackrel{\circ}{Y}$, and $D^{q+1}(\subset Y \times\{1\})$ so that $D^{q} \times I$ is embedded in the piece $\stackrel{\circ}{Y}$. We paste together two copies of $S^{2 k+1} \times Y \times I$ along $S^{2 k+1} \times \stackrel{\circ}{Y}$ by $\widetilde{F}$. The resulting $S^{1}$ manifold $V$ is an $S^{1}$ cobordism between $\widetilde{M}\left(E_{X}([f]), Y, Y\right)$ and $\widetilde{M}\left(E_{S^{q}}([f]), D^{q+1}, D^{q+1}\right)$. Since the $S^{1}$ action on $V$ is free, we have

$$
\sigma\left(g, \widetilde{M}\left(E_{X}([f]), Y, Y\right)\right)-\sigma\left(g, \widetilde{M}\left(E_{S^{q}}([f]), D^{q+1}, D^{q+1}\right)\right)=\operatorname{Sign} V
$$

up to sign. This implies the lemma. Q.E.D.

\section{AdDitivity}

We shall denote $\sigma \cdot E_{X}$ again by $\sigma$. The purpose of this section is to verify Lemma 3.1. $\sigma: D\left(\mathbf{C} P^{k} \times D^{q}\right) \rightarrow F\left(S^{1}\right) / \mathbf{Z}$ is a homomorphism.

Proof. By Lemma 2.1 we may assume $X=S^{q}$. Let $\left[f_{i}\right](i=1,2)$ be elements of $D\left(\mathbf{C} P^{k} \times D^{q}\right)$. Since $f_{i}$ is the identity on the boundary, one can deform $f_{i}$ via isotopy so that $f_{i}$ is the identity on the exterior of $\mathbf{C} P^{k} \times D_{i}$ where $D_{i}$ is a small disk in $D^{q}$. Furthermore we may assume that $D_{1}$ has no intersection with $D_{2}$. 
Set $E_{S^{q}}\left(\left[f_{i}\right]\right)=\left[F_{i}\right] \in D\left(\mathbf{C} P^{k} \times S^{q}\right)$ and let $\widetilde{F}_{i}: S^{2 k+1} \times S^{q} \rightarrow S^{2 k+1} \times S^{q}$ be a lifting of $F_{i}$, which is unique up to $S^{1}$ equivariant isotopy as $H^{1}\left(S^{q} ; \mathbf{Z}\right)=0$. We form a closed $S^{1}$ manifold

$$
\Sigma\left(\left[f_{i}\right]\right)=D^{2 k+2} \times S^{q} \cup S^{2 k+1} \times D^{q+1}
$$

where $D^{2 k+2} \times S^{q}$ and $S^{2 k+1} \times D^{q+1}$ are pasted together by $\widetilde{F}_{i}$ along the boundary and $S^{1}$ acts on $D^{2 k+2}$ linearly extending the free $S^{1}$ action on $S^{2 k+1}$. The action on $\Sigma\left(\left[f_{i}\right]\right)$ is semifree and the fixed point set is $\{0\} \times S^{q}$. It turns out that $\Sigma\left(\left[f_{i}\right]\right)$ is a homotopy $(2 k+q+2)$-sphere.

We regard $\Sigma\left(\left[f_{i}\right]\right)$ as a homotopy $(2 k+q+2)$-sphere with a semifree $S^{1}$ action and a decomposition as in (3.2). The connected sum $\Sigma\left(\left[f_{1}\right]\right) \# \Sigma\left(\left[f_{2}\right]\right)$ can be done equivariantly around fixed points using the decompositions and it is also a homotopy $(2 k+q+2)$-sphere with a semifree $S^{1}$ action and a decomposition. Taking account of decompositions we have

$$
\Sigma\left(\left[f_{1}\right]\right) \# \Sigma\left(\left[f_{2}\right]\right)=\Sigma\left(\left[f_{1} \cdot f_{2}\right]\right) \quad\left(=\Sigma\left(\left[f_{1}\right]\left[f_{2}\right]\right)\right) .
$$

We abbreviate $\widetilde{M}\left(E_{S^{q}}([f]), D^{q+1}, D^{q+1}\right)$ by $\widetilde{M}([f])$. We note that $\widetilde{M}\left(\left[f_{i}\right]\right)$ agrees with the $S^{1}$ manifold obtained from $\Sigma\left(\left[f_{i}\right]\right)$ by doing surgery on the identity map: $D^{2 k+2} \times S^{q} \rightarrow D^{2 k+2} \times S^{q} \subset \Sigma\left(\left[f_{i}\right]\right)$. Therefore $\widetilde{M}\left(\left[f_{1}\right]\left[f_{2}\right]\right)$ is obtained from $\Sigma\left(\left[f_{1}\right]\right) \# \Sigma\left(\left[f_{2}\right]\right)$ in this way.

Consider the equivariant boundary connected sum $\Sigma\left(\left[f_{1}\right]\right) \times I\left\lfloor\Sigma\left(\left[f_{2}\right]\right) \times I\right.$ at the 1-level. This yields an $S^{1}$ cobordism between $\Sigma\left(\left[f_{1}\right]\right) \amalg \Sigma\left(\left[f_{2}\right]\right)$ and $\Sigma\left(\left[f_{1}\right]\right) \# \Sigma\left(\left[f_{2}\right]\right)=\Sigma\left(\left[f_{1}\right]\left[f_{2}\right]\right)$. We attach three copies of the handle $D^{2 k+2} \times D^{q+1}$ to $\Sigma\left(\left[f_{1}\right]\right) \times I$ 七 $\Sigma\left(\left[f_{2}\right]\right) \times I$ via the identity maps from $D^{2 k+2} \times S^{q}$ to $D^{2 k+2} \times$ $S^{q}$ embedded in $\Sigma\left(\left[f_{1}\right]\right) \times\{1\} \# \Sigma\left(\left[f_{2}\right]\right) \times\{1\}, \quad \Sigma\left(\left[f_{1}\right]\right) \times\{0\}$, and $\Sigma\left(\left[f_{2}\right]\right) \times$ $\{0\}$ respectively. This yields a semifree $S^{1}$ cobordism $V$ between $\widetilde{M}\left(\left[f_{1}\right]\right) \amalg$ $\widetilde{M}\left(\left[f_{2}\right]\right)$ and $\widetilde{M}\left(\left[f_{1}\right]\left[f_{2}\right]\right)$. As easily observed the complex normal bundle to $V^{S^{1}}$ is trivial and $\operatorname{Sign} V^{S^{1}}=0$. Hence, similarly to the proof of Lemma 1.1, we have

$$
\sigma\left(, \widetilde{M}\left(\left[f_{1}\right]\right)\right)+\sigma\left(, \widetilde{M}\left(\left[f_{2}\right]\right)\right)=\sigma\left(, \widetilde{M}\left(\left[f_{1}\right]\left[f_{2}\right]\right)\right) \text { in } F\left(S^{1}\right) / \mathbf{Z} .
$$

This proves the lemma as $\sigma([f])=\sigma(, \widetilde{M}([f]))$. Q.E.D.

\section{REVIEW OF THE WORK OF BROWDER-PETRIE}

In this section and the next section we prove the following theorem from which Theorem A follows immediately.

Theorem 4.1. The kernel of $\sigma: D\left(\mathbf{C} P^{k} \times D^{q}\right) \rightarrow F\left(S^{1}\right) / \mathbf{Z}$ is finite.

We need some knowledge about the group $D\left(\mathbf{C} P^{k} \times D^{q}\right)$ for the proof of Theorem 4.1. The group is analysed by Browder-Petrie [BP]. In this section we shall review their work. 
There is a long exact sequence of groups:

$$
\begin{aligned}
\pi_{q+1}\left(G \mathbf{C} P^{k}\right) & \stackrel{\psi}{\rightarrow} h S\left(\mathbf{C} P^{k} \times\left(D^{q+1}, S^{q}\right)\right) \\
& \stackrel{\partial}{\rightarrow} D\left(\mathbf{C} P^{k} \times D^{q}\right) \stackrel{\lambda}{\rightarrow} \pi_{q}\left(G \mathbf{C} P^{k}\right) .
\end{aligned}
$$

Here $G \mathbf{C} P^{k}$ is the identity component of the space of self-maps of $\mathbf{C} P^{k}$ and $h S\left(\mathbf{C} P^{k} \times\left(D^{q+1}, S^{q}\right)\right)$ is the set of equivalence classes of pairs $[Q, h]$ where $Q$ is an oriented smooth manifold and $h:[Q, \partial Q] \rightarrow \mathbf{C} P^{k} \times\left(D^{q+1}, S^{q}\right)$ is a homotopy equivalence preserving orientation and $h \mid \partial Q: \partial Q \rightarrow \mathbf{C} P^{k} \times S^{q}$ is a diffeomorphism. Two pairs $\left[Q_{1}, h_{1}\right]$ and $\left[Q_{2}, h_{2}\right]$ are equivalent if there is a diffeomorphism $d: Q_{1} \rightarrow Q_{2}$ such that the composition $h_{2} \cdot d$ is homotopic to $h_{1}$ relative boundary.

We shall explain the homomorphism $\partial$ and $\lambda$. First we note that $Q$ is diffeomorphic to $\mathbf{C} P^{k} \times D^{q+1}$. This can be seen as follows. Since $h \mid \partial Q$ is a diffeomorphism, $\mathbf{C} P^{k}$ can be embedded in the interior of $Q$ so that the embedding induces a homotopy equivalence and that the normal bundle is trivial. The complement $\stackrel{\circ}{Q}$ of a small open tubular neighborhood of the embedded $\mathbf{C} P^{k}$ in $Q$ turns out to be an $h$-cobordism between $\mathbf{C} P^{k} \times S^{q}$ and $\partial Q=\mathbf{C} P^{k} \times S^{q}$. Hence $\stackrel{\circ}{Q}$ is diffeomorphic to $\mathrm{C} P^{k} \times S^{q} \times I$ by the $h$-cobordism theorem and hence $Q$ is diffeomorphic to $\mathbf{C} P^{k} \times D^{q+1}$. Thus any class in $h S\left(\mathbf{C} P^{k} \times\left(D^{q+1}, S^{q}\right)\right)$ can be represented by a pair $\left[\mathbf{C} P^{k} \times D^{q+1}, h\right]$. Moreover, a similar argument shows that one can choose $h$ so that $h \mid \mathbf{C} P^{k} \times D_{-}^{q}$ is the identity where $D_{-}^{q}$ is the lower hemisphere of $S^{q}$.

With this understood $\partial: h S\left(\mathbf{C} P^{k} \times\left(D^{q+1}, S\right)\right) \rightarrow D\left(\mathbf{C} P^{k} \times D^{q}\right)$ is defined by

$$
\partial\left(\left[\mathbf{C} P^{k} \times D^{q+1}, h\right]\right)=\left[h \mid \mathbf{C} P^{k} \times D_{+}^{q}\right] .
$$

Note that the image of $\partial\left(\left[\mathbf{C} P^{k} \times D^{q+1}, h\right]\right)$ through the homomorphism

$$
E_{S^{q}}: D\left(\mathbf{C} P^{k} \times D^{q}\right) \rightarrow D\left(\mathbf{C} P^{k} \times S^{q}\right)
$$

is nothing but $\left[h \mid \mathbf{C} P^{k} \times S^{q}\right]$.

Let $\rho: \mathbf{C} P^{k} \times D^{q} \rightarrow \mathbf{C} P^{k}$ be the projection. Then $\lambda: D\left(\mathbf{C} P^{k} \times D^{q}\right) \rightarrow$ $\pi_{q}\left(G \mathbf{C} P^{k}\right)$ is defined by

$$
(\lambda([f])(x))(u)=\rho(f(u, x))
$$

where $[f] \in D\left(\mathbf{C} P^{k} \times D^{q}\right), x \in D^{q}$, and $u \in \mathbf{C} P^{k}$.

The linear action of the unitary group $U(k+1)$ on $\mathbf{C} P^{k}$ induces a semihomomorphism $i: U(k+1) / \Delta \rightarrow G \mathbf{C} P^{k}$, where $\Delta$ is the subgroup of $U(k+1)$ consisting of scalar multiples of the identity matrix. It is known that

$$
i_{*}: \pi_{q}(U(k+1) / \Delta) \otimes \mathbf{Q} \rightarrow \pi_{q}\left(G \mathbf{C} P^{k}\right) \otimes \mathbf{Q}
$$

is an isomorphism (see [S] for example). In particular

$$
\pi_{q}\left(G \mathbf{C} P^{k}\right) \otimes \mathbf{Q}= \begin{cases}\mathbf{Q} & \text { if } 3 \leq q \leq 2 k+1(q: \text { odd }), \\ 0 & \text { otherwise }\end{cases}
$$


There is another homomorphism

$$
\mu: \pi_{q}(U(k+1) / \Delta) \rightarrow D\left(\mathbf{C} P^{k} \times D^{q}\right)
$$

defined by

$$
\mu([h])(u, x)=((h(x))(u), x)
$$

where $h:\left(D^{q}, S^{q-1}\right) \rightarrow(U(k+1) / \Delta$, Id $)$. It is easy to see that $\lambda \cdot \mu=i_{*}$. The exact sequence (4.2) together with the above observation shows

Lemma 4.3. The subgroup of $D\left(\mathbf{C} P^{k} \times D^{q}\right)$ generated by the subgroups

$$
\partial\left(h S\left(\mathbf{C} P^{k} \times\left(D^{q+1}, S^{q}\right)\right)\right) \text { and } \mu\left(\pi_{q}(U(k+1) / \Delta)\right)
$$

is of finite index in $D\left(\mathbf{C} P^{k} \times D^{q}\right)$.

\section{Proof of Theorem 4.1}

We abbreviate $\widetilde{M}\left(E_{S^{q}}([f]), D^{q+1}, D^{q+1}\right)$ by $\widetilde{M}$ and let $M$ be the $S^{1}$ orbit space of $\widetilde{M}$. Since the $S^{1}$ action on $\widetilde{M}$ is free, the projection $\widetilde{M} \rightarrow M$ is an $S^{1}$ bundle. Let $\alpha$ be the first Chern class of it. The associated disk bundle $D_{\alpha}$ supports a semifree $S^{1}$ action rotating fibers, the zero section $M$ being the fixed point set. Since $\partial D_{c}=\widetilde{M}$, the Atiyah-Singer invariant $\sigma(g, \widetilde{M})$ can be described using $D_{\alpha}$. In fact, since the normal bundle to $M$ in $D_{\alpha}$ is the complex line bundle with $\alpha$ being the first Chern class, we have

$$
\sigma(g, \widetilde{M})=2^{m} \frac{g e^{\alpha}+1}{g e^{\alpha}-1} L(M)[M]-\operatorname{Sign} D_{\alpha}
$$

where $2 m=2 k+q+1$ and $L$ denotes the Atiyah-Singer $L$-class. Hence, since $\sigma([f])(g)=\sigma(g, \widetilde{M})$ and $\sigma([f])$ is considered in $F\left(S^{1}\right) / \mathbf{Z}$, we have

$$
\sigma([f])=2^{m} \frac{t e^{\alpha}+1}{t e^{\alpha}-1} L(M)[M]
$$

where $t \in F\left(S^{1}\right) / \mathbf{Z}$ is the image of the standard complex 1-dimensional $S^{1}$ representation to $F\left(S^{1}\right) / \mathbf{Z}$.

Expanding $\left(t e^{i x}+1\right) /\left(t e^{i x}-1\right)$ with respect to $e^{i x}-1$, we have

$$
\frac{t e^{\alpha}+1}{t e^{\alpha}-1}=1-2 \sum_{r \geq 0} \frac{t^{r}\left(e^{i x}-1\right)^{r}}{(1-t)^{r+1}} .
$$

It says that $t=1$ is the only pole of $\sigma([f])$.

Lemma 5.3. If $[f] \in \partial\left(h S\left(\mathbf{C} P^{k} \times\left(D^{q+1}, S^{q}\right)\right)\right)$, then the highest degree of the pole of $\sigma([f])$ is at most $k+1$.

Proof. Let $[f]=\partial\left(\left[\mathbf{C} P^{k} \times D^{q+1}, h\right]\right)$. Then $E_{S q}([f])=\left[h \mid \mathbf{C} P^{k} \times S^{q}\right]$ as remarked before. Since $M$ is obtained by pasting together two copies of $\mathbf{C} P^{k} \times D^{q+1}$ along the boundary by $h \mid \mathbf{C} P^{k} \times S^{q}, M$ is homotopy equivalent 
to $\mathbf{C} P^{k} \times S^{q+1}$. Therefore $\alpha^{r}=0$ and hence $\left(e^{\alpha}-1\right)^{r}=0$ for $r>k$. This together with (5.1) and (5.2) implies the lemma. Q.E.D.

Since $\Delta$ is a circle group, the projection map $U(k+1) \rightarrow U(k+1) / \Delta$ induces an isomorphism $\pi_{q}(U(k+1)) \rightarrow \pi_{q}(U(k+1) / \Delta)$ for $q \geq 2$. Suppose $3 \leq$ $q \leq 2 k+1$ ( $q$ : odd $)$. Then $\pi_{q}(U(k+1))$ sits in the stable range; so it is infinite cyclic and is detected by the $(q+1) / 2$ th Chern classes of the complex vector bundles over $S^{q+1}$ corresponding to $\pi_{q}(U(k+1))$. We shall denote by $c([f]) \in H^{q+1}\left(S^{q+1} ; \mathbf{Z}\right)$ the $(q+1) / 2$ th Chern class of

$$
[f] \in \pi_{q}(U(k+1))=\pi_{q}(U(k+1) / \Delta) .
$$

Hereafter we identify $\pi_{q}(U(k+1))$ with a subgroup of $D\left(\mathbf{C} P^{k} \times D^{q}\right)$ via $\mu$ when $3 \leq q \leq 2 k+1$.

Lemma 5.4. Let $3 \leq q \leq 2 k+1$ and $[f] \in \pi_{q}(U(k+1))$. Then the highest degree of the pole of $\sigma([f])$ is at most $k+1+(q+1) / 2$ and the coefficient at the pole of degree $k+1+(q+1) / 2$ is $2^{m+1} c([f])\left[S^{q+1}\right]$ up to sign.

Proof. In this case $M$ is the total space of the complex projective bundle $\pi: M \rightarrow S^{q+1}$ associated with the complex vector bundle over $S^{q+1}$ corresponding to $[f]$. According to Borel-Hirzebruch $[\mathrm{BH}$, p. 516] we have

$$
\alpha^{k+1}+(-1)^{(q+1) / 2} \pi^{*}(c([f])) \alpha^{k+1-(q+1) / 2}=0
$$

and hence

$$
\alpha^{k+(q+1) / 2}=-(-1)^{(q+1) / 2} \pi^{*}(c([f])) \alpha^{k} .
$$

On the other hand (5.1) and (5.2) show that the highest degree of the pole of $\sigma([f])$ is at most $k+1+(q+1) / 2$ and the coefficient at the pole of degree $k+1+(q+1) / 2$ is $2^{m+1} \alpha^{k+(q+1) / 2}[M]$ up to sign. Here we have

$$
\begin{aligned}
\alpha^{k+(q+1) / 2}[M] & = \pm \pi^{*}(c([f])) \alpha^{k}[M] \quad(\text { by }(5.5)) \\
& = \pm c([f])\left[S^{q+1}\right]
\end{aligned}
$$

where the latter identity is the so-called integration along the fiber. This proves the lemma. Q.E.D.

To prove Theorem 4.1 it suffices to show that the kernel of $\sigma$ is finite when $\sigma$ is restricted to the subgroup of $D\left(\mathbf{C} P^{k} \times D^{q}\right)$ which consists of all elements $[f]$ of the form $[f]=\left[f_{1}\right]+\left[f_{2}\right]$ where $\left[f_{1}\right] \in \partial\left(h S\left(\mathbf{C} P^{k} \times\left(D^{q+1}, S^{q}\right)\right)\right)$ and $\left[f_{2}\right] \in \mu\left(\pi_{q}(U(k+1) / \Delta)\right)$. In fact, it suffices to show that each element of this group is of finite order because $D\left(\mathbf{C} P^{k} \times D^{q}\right)$ is finitely generated abelian.

Lemma 5.6. If $\sigma\left(\left[f_{1}\right]+\left[f_{2}\right]\right)=0$, then $\left[f_{2}\right]$ is of finite order.

Proof. We may assume $3 \leq q \leq 2 k+1 \quad(q$ : odd) because otherwise $\pi_{q}(U(k+1) / \Delta)$ is a finite group. Hence we may view $\left[f_{2}\right]$ as an element of $\pi_{q}(U(k+1))=\pi_{q}(U(k+1) / \Delta)$. By Lemma 3.1, we have $\sigma\left(\left[f_{1}\right]\right)+\sigma\left(\left[f_{2}\right]\right)=0$. 
Lemmas 5.3 and 5.4 tell us that the coefficient at the pole of degree $k+1+$ $(q+1) / 2$ is $2^{m+1} c\left(\left[f_{2}\right]\right)\left[S^{q+1}\right]$ up to sign. Since it vanishes, $c\left(\left[f_{2}\right]\right)=0$ and hence $\left[f_{2}\right]=0$. Q.E.D.

To show Theorem 4.1 we need to show only that $\sigma$ has a finite kernel when restricted to $\partial\left(h S\left(\mathbf{C} P^{k} \times\left(D^{q+1}, S^{q}\right)\right)\right)$, or any element in this kernel is of finite order. Remember that $M$ is then homotopy equivalent to $\mathbf{C} P^{k} \times S^{q+1}$.

Lemma 5.7. Let $[f] \in \partial\left(h S\left(\mathbf{C} P^{k} \times\left(D^{q+1}, S^{q}\right)\right)\right)$. If $\sigma([f])=0$, then the total Pontrjagin class $p(M)$ of $M$ is of the same form as $\mathbf{C} P^{k} \times S^{q+1}$, i.e. $p(M)=$ $\left(1+\alpha^{2}\right)^{k+1}$.

Proof. Since $M$ is homotopy equivalent to $\mathbf{C} P^{k} \times S^{q+1}$, one can express

$$
L(M)=u(\alpha)+v(\alpha) \beta
$$

where $u(\alpha)$ and $v(\alpha)$ are polynomials of $\alpha$ of degree at most $k$ and $\beta$ is a generator of $H^{q+1}(M)$ corresponding to the factor $S^{q+1}$.

Remember that $M=\mathbf{C} P^{k} \times D^{q+1} \cup \mathbf{C} P^{k} \times D^{q+1}$; so $\mathbf{C} P^{k}$ is naturally embedded in $M$ with the trivial normal bundle. This means that the restriction of $L(M)$ to the embedded $\mathbf{C} P^{k}$ is of the same form as $\mathbf{C} P^{k}$. Hence $u(\alpha)$ is determined. The identities (5.1) and (5.2) tell us that $\sigma([f])$ determines the values $\alpha^{r} L(M)[M]$ for $0 \leq r \leq k$, which determine $v(\alpha)$, in fact $v(\alpha)=0$.

It is known that the total $L$-classes determine the total Pontrjagin classes and vice versa. Consequently $\sigma([f])$ determines $p(M)$. Since $p(M)=\left(1+\alpha^{2}\right)^{k+1}$ is a solution of the equation $\sigma([f])=0$, the lemma follows. Q.E.D.

Let $[f]=\partial\left(\left[Q_{f}, h_{f}\right]\right)$. Remember that we may assume $Q_{f}=\mathbf{C} P^{k} \times D^{q+1}$. Since $\pi_{q+1}\left(G \mathbf{C} P^{k}\right)$ is finite as $q+1$ is even, the map $\partial$ has finite kernel by (4.2). Therefore to complete our proof of Theorem 4.1, it suffices to show that $\left[Q_{f}, h_{f}\right]$ is of finite order. This we shall do now. Petrie [P] defined a map

$$
\gamma: h S\left(\mathbf{C} P^{k} \times\left(D^{q+1}, S^{q}\right)\right) \rightarrow h S\left(\mathbf{C} P^{k} \times S^{q+1}\right)
$$

as follows: $\gamma([Q, h])=[\gamma(Q), \gamma(h)]$ where $\gamma(Q)$ is the manifold obtained by pasting together $Q$ and $\mathbf{C} P^{k} \times D^{q+1}$ along the boundary by $h \mid \partial Q$, and $\gamma(h)|Q=h, \quad \gamma(h)| \mathbf{C} P^{k} \times D^{q+1}$ is the identity. We note that $M=\gamma\left(Q_{f}\right)$. By Lemma $5.7 \gamma\left(Q_{f}\right)$ has the Pontrjagin classes of the same form as $\mathbf{C} P^{k} \times S^{q+1}$. This fact seems to imply that $\left[\gamma\left(Q_{f}\right), \gamma\left(h_{f}\right)\right]$ is of finite order in $h S\left(\mathbf{C} P^{k} \times S^{q+1}\right)$. However this argument does not work because $h S\left(\mathbf{C} P^{k} \times S^{q+1}\right)$ does not admit a natural group structure. 
To avoid this trouble we shall consider the following commutative diagram of surgery exact sequences:

$$
h S\left(\mathbf{C} P^{k} \times\left(D^{q+1}, S^{q}\right)\right) \stackrel{\eta}{\longrightarrow}\left[\mathbf{C} P^{k} \times D^{q+1} / \mathbf{C} P^{k} \times S^{q}, G / O\right]
$$

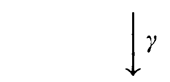

0

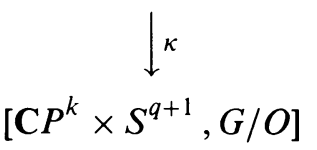

Here $G / O$ is the homotopy fiber of the classifying map $B O \rightarrow B G$ ( $B G$ is the classifying space of stable spherical fibrations), and $[A, G / O]$ is the set of homotopy classes of continuous maps from $A$ to $G / O$. In fact, induced from the $H$-space structure of $G / O$, the set $[A, G / O]$ forms an abelian group. The vertical map $\kappa^{*}$ is a homomorphism induced from the quotient map $\kappa: \mathbf{C} P^{k} \times$ $S^{q+1} \rightarrow \mathbf{C} P^{k} \times D^{q+1} / \mathbf{C} P^{k} \times S^{q}$. It is known that $\eta$ is a homomorphism.

Let $A$ be a finite $\mathrm{CW}$-complex. The inclusion map $j: G / O \rightarrow B O$ induces a homomorphism $j_{*}:[A, G / O] \rightarrow[A, B O]=\widetilde{K} O(A)$ and there is a functor ph (called Pontrjagin character) from $\widetilde{K} O(A)$ to $\widetilde{H}^{4^{*}}(A ; \mathbf{Q})$. It is well known that

$j_{*}$ and $\mathrm{ph}$ are both isomorphisms when tensored by $\mathbf{Q}$.

As easily checked $\kappa^{*}: \widetilde{H}^{4^{*}}\left(\mathbf{C} P^{k} \times D^{q+1} / \mathbf{C} P^{k} \times S^{q} ; \mathbf{Q}\right) \rightarrow \widetilde{H}^{4^{*}}\left(\mathbf{C} P^{k} \times S^{q+1} ; \mathbf{Q}\right)$ is injective. Hence it follows from (5.9) that $\kappa^{*}$ in the diagram (5.8) has finite kernel. In the sequel it suffices to show that $\eta^{\prime}\left(\left[\gamma\left(Q_{f}\right), \gamma\left(h_{f}\right)\right]\right)$ is of finite order since $\eta$ is a monomorphism.

It is also known that

$$
\begin{aligned}
\mathrm{ph} \cdot j_{*} & \cdot \eta^{\prime}\left(\left[\gamma\left(Q_{f}\right), \gamma\left(h_{f}\right)\right]\right) \\
& =\left(\gamma\left(h_{f}\right)^{*}\right)^{-1} \operatorname{ph}\left(\gamma\left(Q_{f}\right)\right)-\operatorname{ph}\left(\mathbf{C} P^{k} \times S^{q+1}\right)
\end{aligned}
$$

where $\operatorname{ph}(B)$ denotes the Pontrjagin character of the tangent bundle of a manifold $B$. Since $p\left(\gamma\left(Q_{f}\right)\right)=\left(1+\alpha^{2}\right)^{k+1},\left(\gamma\left(h_{f}\right)^{*}\right)^{-1} p\left(\gamma\left(Q_{f}\right)\right)=p\left(\mathbf{C} P^{k} \times S^{q+1}\right)$ and hence the right-hand side of (5.10) is zero. Thus $\eta^{\prime}\left(\left[\gamma\left(Q_{f}\right), \gamma\left(h_{f}\right)\right]\right)$ is of finite order by (5.9). Q.E.D.

\section{REFERENCES}

[AS] M. F. Atiyah and I. M. Singer, The index of elliptic operators. III, Ann. of Math. 87 (1968), 546-604.

[BH] A. Borel and F. Hirzebruch, Characteristic classes and homogeneous spaces. I, Amer. J. Math. 80 (1958), 459-538.

[B] W. Browder, Diffeomorphisms of 1-connected manifolds, Trans. Amer. Math. Soc. 128 (1967), 155-163.

[BP] W. Browder and T. Petrie, Diffeomorphisms of manifolds and semi-free actions on homotopy spheres, Bull. Amer. Math. Soc. 77 (1971), 160-163.

[C] J. Cerf, La stratification naturelle des espaces de fonctions différentiables réelles et le théorème de la pseudo-isotopie, Inst. Hautes Études Sci. Publ. Math. 39 (1970). 
[KM] M. Kervaire and J. Milnor, Groups of homotopy spheres. I, Ann. of Math. 77 (1963), 504-537.

[L] J. Levine, Self-equivalences of $S^{r} \times S^{k}$, Trans. Amer. Math. Soc. 143 (1969), 523-543.

[P] T. Petrie, The Atiyah-Singer invariant, the Wall groups $L_{n}(\pi, 1)$, and the function $\left(e^{x}+\right.$ 1)/(te -1$)$, Ann. of Math. 92 (1970), 174-187.

[S] S. Sasao, The homotopy of $\operatorname{Map}\left(\mathbf{C} P^{m}, \mathbf{C} P^{n}\right)$, J. London Math. Soc. 8 (1974), 193-197.

Department of Mathematics, Osaka City University, Sugimoto, Sumiyoshi-Ku, Osaka 558, JAPAN 